\title{
Nonmonotonous temperature dependence of the initial decoherence in quantum dots
}

\author{
A. Vagov \\ Physics Department, University of Antwerp, Universiteitsplein 1, 2610 Wirlijk, Antwerp, Belgium \\ V. M. Axt and T. Kuhn \\ Institut für Festkörpertheorie, Universität Münster, Wilhelm-Klemm Str. 10, 48149 Münster, Germany \\ W. Langbein, P. Borri, and U. Woggon \\ Experimentelle Physik EIIb, Universität Dortmund, Otto-Hahn Str. 4, 44227 Dortmund, Germany
}

(Received 19 August 2004; published 11 November 2004)

\begin{abstract}
We have analyzed the initial decoherence of strongly confined semiconductor quantum dots by performing four-wave-mixing experiments and comparing with theory. The measurements are in quantitative agreement with analytical results accounting for the pure dephasing induced by acoustic phonons. The experiments confirm the recent prediction of an unusual nonmonotonous temperature dependence of the initial decoherence time.

DOI: 10.1103/PhysRevB.70.201305

PACS number(s): 78.67.Hc, 63.20.Kr, 63.22.+m, 78.47.+p
\end{abstract}

Semiconductor quantum dots (QDs) attract attention as they resemble macroatoms with externally controllable properties in a solid-state environment. These features make them relevant for optoelectronic applications such as lasers ${ }^{1}$ or single photon sources. ${ }^{2}$ QDs are also discussed as key ingredients of quantum-information processing ${ }^{3,4}$ or quantum encryption devices. ${ }^{5}$ Decoherence is a decisive limiting factor for the operation of most existing or potential applications, which explains the broad interest in this issue. ${ }^{6-13}$ In general, coherence is lost due to couplings of the discrete QD excitations to the environment provided, e.g., by phonons, ${ }^{6-11}$ charge fluctuations in the surrounding material, ${ }^{13}$ or by the radiation field. ${ }^{12}$ Many corresponding studies ${ }^{6-12,14,15}$ have revealed that the decoherence of QD excitations constitutes a nontrivial dynamical phenomenon which is by itself of physical interest, in addition to its importance for device applications. Particularly striking is the behavior of selfassembled QDs. ${ }^{8,9}$ In such systems the decoherence of the optical polarization takes place in two stages that, at not too high temperatures, are well distinguished, ${ }^{8,15}$ namely: an initial rapid decay which is nonexponential and typically ends after a few picoseconds, and a subsequent almost exponential decay on a much longer time scale. The rather short time scale for the initial decay puts a constraint for device operations, but on the other hand, the coherence is only partly lost by a temperature-dependent amount. ${ }^{15}$ To obtain a clear picture of the limitations introduced by the initial decoherence it is thus relevant to quantify the initial decoherence time as well as the degree of initially lost coherence.

In self-assembled QDs that exhibit strong electronic confinement, phonon-assisted transitions between separated electronic levels are limited. Thus, elastic scattering of acoustic phonons can become an important decoherence mechanism, a process commonly referred to as pure dephasing. ${ }^{6,10,11}$ Recent results indicated the important role of pure dephasing for the decoherence of QD systems. For example, photoluminescence (PL) spectra detected from individual QDs ${ }^{11}$ comprise a sharply peaked Lorentzian zerophonon line (ZPL) superimposed on a broadband back- ground that is typically much wider than the radiative linewidth. The broad background reflects the rapid initial decay observed in time domain experiments. Many of the decoherence channels available in QDs correspond to rather slow processes, e.g., the radiative decay or charge fluctuations, which cannot explain the rapid initial decay. In contrast, calculations within the pure dephasing model were able to reproduce the observed order of magnitude of the background width as well as the nontrivial shape of the spectra. ${ }^{11}$ Information on the dephasing dynamics can be accessed by four-wave-mixing (FWM) measurements on QD ensembles. ${ }^{6-9,16}$ Here, it is used that FWM signals form photon echoes in inhomogeneously broadened ensembles since the nonlinear polarization contains a phase conjugated part. The occurrence of an echo indicates a rephasing of the transitions from different ensemble members and thus its peak value-which for sharp echoes is proportional to the time integral-reflects the polarization dynamics associated with the homogeneous dephasing. It has been found that the pure dephasing contribution to the decoherence qualitatively reproduces the observed initial decay provided the nonlinear character of the signal is correctly accounted for. ${ }^{15}$ From the good qualitative agreement between model calculations concentrating on pure dephasing with measured single-dot PL spectra and FWM transients, it was concluded that pure dephasing yields a major contribution to the initial decoherence of strongly confined QDs. The model calculations additionally predicted that the initial decay time should depend on the temperature in a nonmonotonous way: at low temperatures it increases with rising temperature. ${ }^{15}$ Conversely, it is known experimentally ${ }^{8}$ that the long-time decay follows - in agreement with corresponding theories ${ }^{17-19}$ - the more usual behavior of a monotonously decreasing decay time with rising temperature. Testing experimentally the prediction of the nonmonotonous temperature dependence of the initial decoherence time is therefore not only interesting by itself, but also provides a criterium to decide whether the physical nature of the initial decay mechanism is correctly assigned to pure dephasing. 


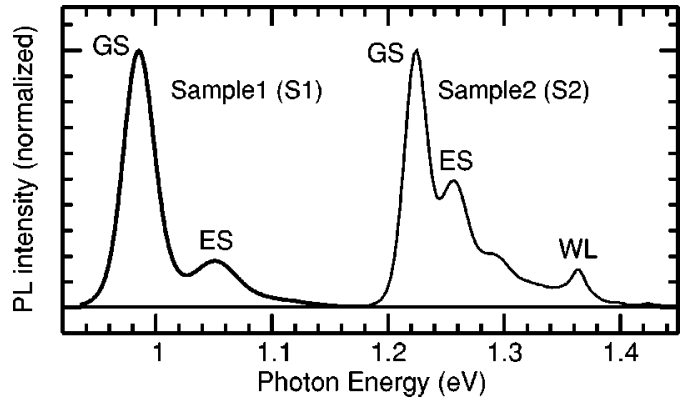

FIG. 1. Nonresonantly excited PL spectra at low excitation intensities at $300 \mathrm{~K}$ for the nonannealed (sample 1) and the annealed sample (sample 2).

In this Rapid Communication we present an experimental and theoretical analysis of the temperature dependence of the initial decoherence time and the fraction of initially lost coherence. We have performed FWM experiments on InGaAs QD ensembles in the temperature range from 5 to $100 \mathrm{~K}$. The measured data are compared with analytical results for the corresponding pure dephasing contribution. The measurements exhibit the predicted nonmonotonous temperature dependence of the initial decoherence time, and quantitatively agree with the theory.

The samples are planar wafers containing InAs/GaAs self-assembled QDs grown on a GaAs(100) substrate using molecular-beam epitaxy. Ten stacked layers of QDs were grown separated by 100-nm GaAs spacing layers and with $2-3 \times 10^{10} \mathrm{~cm}^{-2}$ areal density, i.e., quantum-mechanically uncoupled. Sample 1 was obtained by deposition of a nominal coverage of 2.1 monolayers of InAs at a substrate temperature of $515^{\circ} \mathrm{C}$ resulting in the formation of InAs islands. Sample 2 was obtained by a rapid thermal annealing of a piece of sample 1 for $30 \mathrm{~s}$ at $860{ }^{\circ} \mathrm{C}$. In doing so, the depth of the in-plane confining potential is decreased due to the thermally induced In diffusion. ${ }^{20}$ Figure 1 shows roomtemperature PL spectra of the two samples. The two peaks correspond to the ground (GS) and first excited (ES) excitonic states. The blue shift of the GS transition of sample 2 due to thermal annealing also reduced the energy distance of the GS to the wetting layer (WL) from 330 to $140 \mathrm{meV}$.

We performed two-beam transient FWM measurements in transmission geometry using a directional selection scheme together with a heterodyne detection. ${ }^{21}$ The Fourierlimited excitation pulses of $150-$ fs duration at $76-\mathrm{MHz}$ repetition rate were in resonance with the center of the inhomogeneously broadened GS transitions, and co-circularly polarized to suppress biexcitonic contributions. ${ }^{8}$ In heterodyne detection we measure the FWM field amplitude using its interference with the field of a reference pulse. The samples were antireflection coated to reduce multiple reflections and were held in a cryostat at temperatures varying from 5 to $100 \mathrm{~K}$.

The starting point for our theoretical discussion is the model for phonon-induced pure dephasing of optically excited QDs presented in Refs. 10 and 11. The electronic degrees of freedom of a QD are represented as a two-level system coupled to laser light via the dipole coupling, and to acoustic phonons via the deformation potential coupling.
Other phonon couplings were included in the theory, but turned out to be of minor importance for our QD samples ${ }^{10}$ and will thus be neglected. Without the light-field coupling the model reduces to the well-studied independent Boson model. Recently, we have derived analytical solutions for the dynamics of all elements of the electronic and phononic density matrices in the limit of $\delta$-pulse excitation by an arbitrary pulse sequence. ${ }^{14}$ This limit is applicable to our experiments as we use pulses that are much shorter than the typical time scale for the intrinsic system evolution. The solution is nonperturbative with respect to both the exciton-light and the exciton-phonon coupling and is therefore not limited to low excitation densities or weak phonon couplings. For the present case of a two-pulse FWM signal emitted from a strongly inhomogeneously broadened sample the time-integrated ensemble averaged polarization reads ${ }^{15}$

$$
P=F \exp \left[-8 \sum_{\mathbf{q}}\left|\gamma_{\mathbf{q}}\right|^{2}\left(1+2 N_{\mathbf{q}}\right) \sin ^{4}\left(\frac{\omega_{\mathbf{q}} \tau}{2}\right)\right],
$$

where $\tau>0$ is the relative time delay between the exciting pulses, $N_{\mathbf{q}}$ is the thermal phonon occupation at temperature $T$ of a phonon mode with wave vector $\mathbf{q}$, frequency $\omega_{\mathbf{q}}$, and $\gamma_{\mathbf{q}} \equiv\left(g_{\mathbf{q}}^{e}-g_{\mathbf{q}}^{h}\right) / \omega_{\mathbf{q}}$. It is sufficient for the present type of materials to assume a linear dispersion $\omega_{\mathbf{q}}=c|\mathbf{q}|$ (cf. Ref. 10), where $c$ is the sound velocity. The factor $F$ is independent of $T$ and $\tau$. It contains the information on the pulse area dependence which is not important here. Finally, the deformation potential coupling to electrons (holes) is given by

$$
g_{\mathbf{q}}^{\mathrm{e}(\mathrm{h})}=\frac{q D^{\mathrm{e}(\mathrm{h})}}{\sqrt{2 V \rho \hbar \omega_{\mathbf{q}}}} \int\left|\psi_{\mathrm{e}(\mathrm{h})}(\mathbf{r})\right|^{2} e^{i \mathbf{r q}} d^{3} \mathbf{r},
$$

where $\rho$ is the density of the material, $D^{\mathrm{e}(\mathrm{h})}$ is the deformation potential constant, and $\psi_{\mathrm{e}(\mathrm{h})}(\mathbf{r})$ is the wave function of the confined particle. In the absence of precise information about the QD structure we assume a parabolic nonisotropic confinement potential, rotationally symmetric around the growth direction axis $z$. The ground-state wave function for such a model is a Gaussian with two parameters: the lateral diameter $a_{\mathrm{e}(\mathrm{h})}$ and the full $z$-width $b_{\mathrm{e}(\mathrm{h})}$, where the electron (hole) density has decreased by $1 / e$ from its peak value. The material parameters entering Eqs. (1) and (2) are available from the literature 22 except for the confinement lengths $a_{\mathrm{e}(\mathrm{h})}$ and $b_{\mathrm{e}(\mathrm{h})}$, which are thus the only adjustable values depending on the individual sample. Assuming equal potential shapes for electrons and holes allows us to express the localization ratios $a_{\mathrm{h}} / a_{\mathrm{e}}$ and $b_{\mathrm{h}} / b_{\mathrm{e}}$ in terms of the electron/hole mass ratios for the in-plane and growth direction masses, leaving $a \equiv a_{\mathrm{e}}$ and $b \equiv b_{\mathrm{e}}$ as the only free parameters of the theory.

As discussed in Ref. 8, the measured FWM signal is a sharp photon echo in real time. From the time-resolved data we have extracted the time-integrated FWM amplitudes plotted in Fig. 2 as a function of the delay time $\tau$. To facilitate the comparison of the initial decay properties disentangled from the long-time behavior, we have normalized the curves by first fitting an exponential decay $A_{0} \exp (-\gamma \tau)$ to the data 


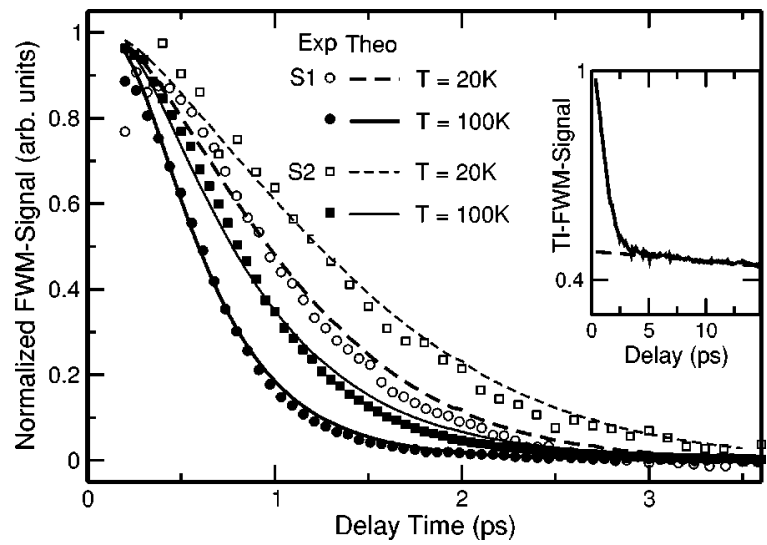

FIG. 2. Time-integrated FWM amplitudes normalized as described in the text plotted vs delay time $\tau$ for both samples $(S 1 / S 2)$ at temperatures 20 and $100 \mathrm{~K}$. The symbols represent the experiment and lines the theory with $a=12.6 \mathrm{~nm}, b=2.4 \mathrm{~nm}$ for sample 1 and $a=16 \mathrm{~nm}, b=2.4 \mathrm{~nm}$ for sample 2 . The inset shows the nonnormalized FWM amplitude for sample 2 at $20 \mathrm{~K}$ and the extracted long-time behavior (dashed).

for delay times $\tau>6 \mathrm{ps}$. The raw data are then divided by this exponential, the asymptotic value is subtracted, and the data are normalized to their maximum value. ${ }^{23}$ An example for the raw data is shown in the inset of Fig. 2. The measured signals (symbols in Fig. 2) exhibit a smooth nonexponential decay on a picosecond time scale. Unlike previous results obtained with linearly polarized light ${ }^{8}$ no beats are detected on this time scale, demonstrating that biexcitonic contributions are suppressed. This allows for a precise quantitative analysis of the phonon-induced dephasing also for low temperatures, at which exciton-biexciton beats would dominate the initial dynamics.

Also shown in Fig. 2 are curves calculated according to Eq. (1), where the electronic confinement lengths $a$ and $b$ have been used as independent fitting parameters keeping the electron/hole localization ratios at the values determined by the mass ratios: $a_{\mathrm{h}} / a_{\mathrm{e}}=0.87, b_{\mathrm{h}} / b_{\mathrm{e}}=0.65$. The fits result in the values $a=12.6 \mathrm{~nm}, b=2.4 \mathrm{~nm}$ for sample 1 and $a=16 \mathrm{~nm}, b=2.4 \mathrm{~nm}$ for sample 2 , in agreement with the expectation that the annealing should result in increased lateral sizes of the wave function. The value of $a$ could alternatively be adjusted by demanding that the ES-GS energy separation observed in the PL spectra (see Fig. 1) is reproduced by the oscillator confinement model (only $a$ can be obtained in this way because the energy difference is due to a change of the in-plane wave function). This procedure yields $a_{\mathrm{e}}=11.8$ and $16.7 \mathrm{~nm}$ for samples 1 and 2, respectively, consistent with the values obtained from fitting the FWM data. The comparison between experiment and theory in Fig. 2 demonstrates that the characteristic nonexponential shape of the FWM transients is well represented by Eq. (1).

To quantify the initial loss of coherence we have determined the fraction by which the signal has dropped after the initial decay is completed (see inset of Fig. 2), which allows one to infer the weight of the ZPL in the homogeneous line shape. ${ }^{14}$ Note that in the photon echo polarization dynamics, the ZPL weight is the third power of the ZPL weight in the

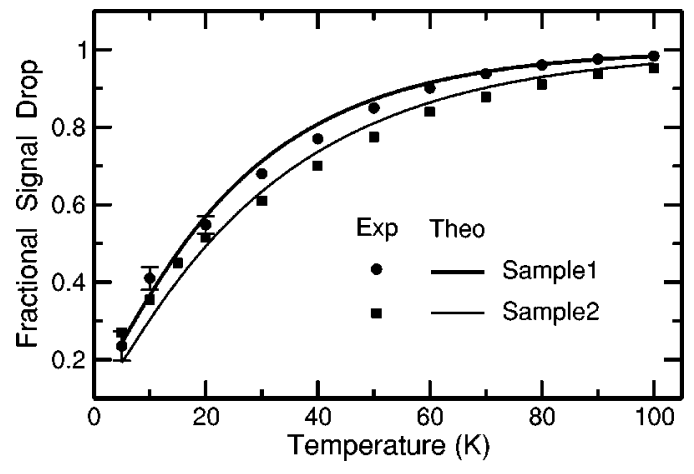

FIG. 3. Fractional signal drop after $\tau=6 \mathrm{ps}$ vs temperature: experiment (symbols) and theory (lines) calculated with the same parameters as in Fig. 2. Error bars represent the statistical error of the extraction method (Ref. 23).

linear polarization, ${ }^{14}$ which was not considered in the analysis of Ref. 8. Experimental (symbols) and theoretical (lines) results for the fractional drop are plotted in Fig. 3 versus temperature. Even at low $T$ the signals are found to drop by more than 20\%. This fraction increases monotonously with rising $T$ for both samples.

A measure for the time scale on which the initial decoherence takes place is the delay time $\tau_{\text {initial }}$ at which the normalized signal (see Fig. 2) has dropped to half of its initial amplitude. The extracted $\tau_{\text {initial }}$ are plotted in Fig. 4(a) versus temperature. ${ }^{23}$ The figure confirms the expected tendency of a faster decoherence for smaller extensions of the exciton wave function. The most striking feature is however that $\tau_{\text {initial }}$ clearly is a nonmonotonous function of $T$, increasing at low and decreasing at higher temperatures. Its maximum is reached at slightly different $T$ values for different samples. The observed nonmonotonous $T$ dependence of $\tau_{\text {initial }}$ can be explained as follows: ${ }^{15}$ At $T=0$ the effective phonon coupling, i.e., the angle average of $q^{2}\left|\gamma_{\mathbf{q}}\right|^{2}\left(1+2 N_{\mathbf{q}}\right)$, peaks at a finite $q$ value $q_{\max }$ corresponding to a phonon frequency $\omega_{q \max }$. This peak in the phonon sideband leads to oscillations of ultrafast signals with a frequency $\omega_{q \max }$ which are overdamped because the width of the peak is of the order of

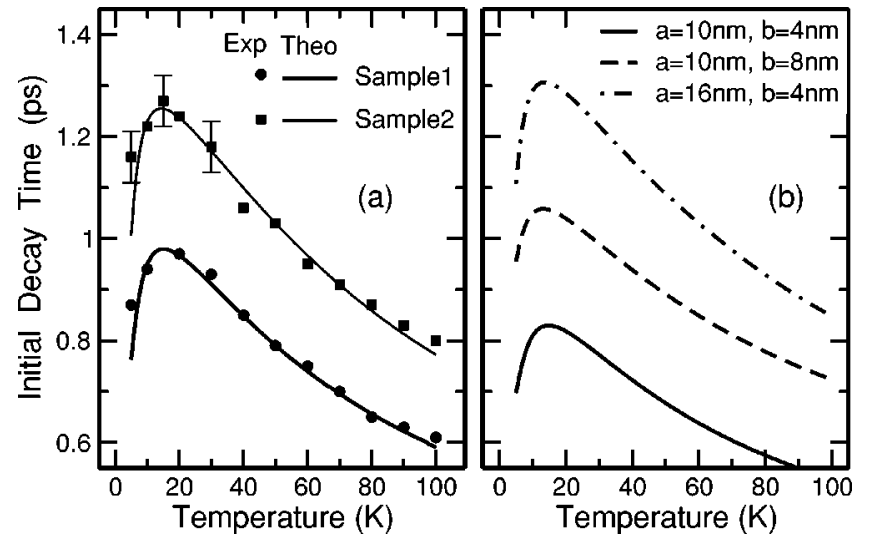

FIG. 4. Initial decoherence time vs temperature: (a) experiment (symbols) and theory (lines) using the same parameters as in Fig. 2; (b) calculations for different wave-function sizes. Error bars represent the statistical error of the extraction method (Ref. 23). 
$\omega_{q \max }$ and thus the signals describe a pure decay that is completed after about half a period of this oscillation. ${ }^{15}$ With increasing $T$ the peak broadens and moves towards $q_{\max }=0$ (i.e., $\omega_{q \max } \rightarrow 0$ ) due to the singularity of the Bose distribution $N_{\mathbf{q}}$. At low temperatures the half period $\pi / \omega_{q \max }$ increases faster with rising temperature than the width of the sideband leading to the unusual rise of $\tau_{\text {initial }}$ at low $T$ values. The high-temperature behavior is dominated by the effective damping which reflects the total width of the coupled phonon sidebands. The latter increases with temperature, resulting in the observed decrease of $\tau_{\text {initial }}{ }^{15,18}$

Finally, Fig. 4(b) illustrates the influence of the size parameters $a$ and $b$. Interestingly, essential features of the curves cannot be changed by variations of $a$ or $b$. In particular, the nonmonotonous $T$ dependence of $\tau_{\text {initial }}$ is always found. Moreover, the parameters have little impact on the shape of the curves and the position of the maximum. Thus, the predominant effect of size variations is a shift of the overall time scale.
In conclusion, we have measured the temperature dependence of the initial decoherence in strongly confined QDs by FWM, and compared the result with a theoretical model of pure dephasing by acoustic phonons. While the total coherence loss rises monotonously with temperature, the decoherence time shows an unusual nonmonotonous temperature dependence. A quantitative agreement between measurement and a theory of pure dephasing by acoustic phonon interactions is found. This unusual behavior of the initial decoherence is expected to be generally present in localized electronic excitations that couple to a continuum of vibrational modes. Couplings of this type exist essentially in every semiconductor material, but can be found in even larger classes of solid-state systems.

This work was supported by the DFG (GRK726) and A.V. is supported by DWTC. The samples were grown and annealed by V. Stavarache and D. Reuter in the group of A.D. Wieck at the University of Bochum, Germany.
${ }^{1}$ D. Bimberg, M. Grundmann, and N. N. Ledentsov, Quantum Dot Heterostructures (Wiley, Chichester, 1998).

${ }^{2}$ P. Michler, A. Kiraz, C. Becher, W. V. Schoenfeld, P. M. Petroff, L. D. Zhang, E. Hu, and A. Imamoglu, Science 290, 2282 (2000).

${ }^{3}$ E. Biolatti, R. C. Iotti, P. Zanardi, and F. Rossi, Phys. Rev. Lett. 85, 5647 (2000).

${ }^{4}$ X. Q. Li, Y. W. Wu, D. Steel, D. Gammon, T. H. Stievater, D. S. Katzer, D. Park, C. Piermarocchi, and L. J. Sham, Science 301, 809 (2003).

${ }^{5}$ R. M. Stevenson, R. M. Thompson, A. J. Shields, I. Farrer, B. E. Kardynal, D. Ritchie, and M. Peppes, Phys. Rev. B 66, 081302(R) (2002).

${ }^{6}$ X. D. Fan, T. Takagahara, J. E. Cunningham, and H. L. Wang, Solid State Commun. 108, 857 (1998).

${ }^{7}$ P. Borri W. Langbein, J. Mørk, J. M. Hvam, F. Heinrichsdorff, M. M. Mao, and D. Bimberg, Phys. Rev. B 60, 7784 (1999).

${ }^{8}$ P. Borri, W. Langbein, S. Schneider, U. Woggen, R. L. Sellin, D. Ouyang, and D. Bimberg, Phys. Rev. Lett. 87, 157401 (2001).

${ }^{9}$ D. Birkedal, K. Leosson, and J. M. Hvam, Phys. Rev. Lett. 87, 227401 (2001).

${ }^{10}$ B. Krummheuer, V. M. Axt, and T. Kuhn, Phys. Rev. B 65, 195313 (2002).

${ }^{11}$ L. Besombes, K. Kheng, L. Marsal, and H. Mariette, Phys. Rev. B 63, 155307 (2001).

${ }^{12}$ M. Bayer and A. Forchel, Phys. Rev. B 65, 041308(R) (2002).

${ }^{13}$ T. Itakura and Y. Tokura, Phys. Rev. B 67, 195320 (2003).
${ }^{14}$ A. Vagov, V. M. Axt, and T. Kuhn, Phys. Rev. B 66, 165312 (2002).

${ }^{15}$ A. Vagov, V. M. Axt, and T. Kuhn, Phys. Rev. B 67, 115338 (2003).

${ }^{16}$ L. V. Dao, M. Lowe, P. Hannaford, H. Makino, T. Takai, and T. Yao, Appl. Phys. Lett. 81, 1806 (2002).

${ }^{17}$ T. Takagahara, Phys. Rev. B 60, 2638 (1999).

${ }^{18}$ X.-Q. Li and Y. Arakawa, Phys. Rev. B 60, 1915 (1999).

${ }^{19}$ A. V. Uskov, A. P. Jauho, B. Tromborg, J. Mørk, and R. Lang, Phys. Rev. Lett. 85, 1516 (2000).

${ }^{20}$ S. Fafard and C. N. Allen, Appl. Phys. Lett. 75, 2374 (1999).

${ }^{21}$ P. Borri W. Langbein, U. Woggen, H. Schwab, U. Bayer, S. Fafard, Z. Wasilewoki, and P. Hawrylak, Phys. Rev. Lett. 91, 267401 (2003).

${ }^{22}$ Most uncertain are the absolute values of $D^{\mathrm{e}(\mathrm{h})}$ (only $\left|D^{\mathrm{e}}-D^{\mathrm{h}}\right|$ is well known). Here we have used $D^{\mathrm{e}}=7 \mathrm{eV}$ and $D^{\mathrm{h}}=-3.5 \mathrm{eV}$. All other material parameters have well-established standard values listed, e.g., in Ref. 10.

${ }^{23}$ For $\tau<0.2$ ps the FWM from the QDs is covered by nonresonant FWM of the GaAs substrate, and is also modified by the finite rise time of the photon echo. In order to determine the correct initial amplitude, we have fitted the experimental data for $0.2 \mathrm{ps}<\tau<0.8 \mathrm{ps}$ by a Gaussian centered at $\tau=0$. Using a series of other bell-shaped fitting functions we have verified that essentially the same behaviors are obtained for the results in Figs. 3 and 4 . 